ARTÍCULOS ORIGINALES Rev Chil Salud Pública 2014; Vol 18 (1): 61-71

\section{INFLUENCIA DE APOYO SOCIAL Y USO DE SERVICIOS DE SALUD SOBRE LA DEPRESIÓN EN CUIDADORES DE ADULTOS MAYORES EN ZONA RURAL}

\author{
INFLUENCE OF SOCIAL SUPPORT AND USE OF HEALTH SERVICES ON \\ DEPRESSION AMONG CARETAKERS OF SENIOR CITIZENS IN RURAL AREAS
}

\section{RESUMEN}

Objetivos: Investigar la influencia de las redes sociales, apoyo social y uso de servicios de salud sobre la depresión en cuidadores de adultos mayores rurales.

Sujetos: 100 cuidadores rurales proveedores de cuidados para adultos mayores.

Método: Estudio transversal con muestreo aleatorio estratificado; se administra a los cuidadores la escala de apoyo social (EAS), de redes sociales (ERS) y de depresión (CES-D).

Resultados: Los cuidadores se dividieron en dos grupos según proveyeran cuidados a adultos mayores sanos (AMS) o enfermos (AME). Los cuidadores para AMS y AME fueron esposa (28 y 43\%), hijos y nueras (51 y 40\%) y nietos (2 y 7,5\%) respectivamente. Los tres servicios de salud más usados por cuidadores fueron visita médica (65,2\%), ayuda de familiares/amigos (41,2\%), curadores alternativos o medicinas no tradicionales (31,9\%). Hubo diferencias significativas entre cuidadores de adultos mayores sanos y enfermos en depresión ( $t=3.195, p<0.01)$ y ERS $(t=1.955$, $p<0.05)$. La depresión se asocia con edad de cuidadores $(\beta=2.332)$, ingresos $(\beta=-0.325)$, tiempo de cuidados $(\beta=0.253)$, ERS $(\beta=-1.984, p=0.001)$ y género del adulto mayor $(\beta=0.143, p<0.003)$.

Discusión: La familia es la principal fuente de cuidados en medios rurales de menores ingresos socioeconómicos, la esposa e hijas ocupan roles centrales, usando recursos médicos y no tradicionales. La depresión de cuidadores se asocia con características de adultos mayores, siendo la disponibilidad de fuentes de apoyo un mediador potencial.

Conclusión: La disponibilidad de apoyos sociales y uso de servicios de salud son importantes para cuidadores rurales, como recursos para disminuir la severidad y presencia de depresión

Palabras clave: adultos mayores, cuidadores, rural, medios socio-económicos, apoyo social, recursos sociales, depresión. 


\section{ABSTRACT}

Goals: Investigate the influence of social networks, social support and health service used for depression among rural elder care providers.

Subjects: 100 rural elder care providers.

Method: Cross sectional study with a randomized stratified sample, using the elder care providers' social support scale (EAS), social networks (ERS), and depression (CES-D).

Results: Elder care providers were divided into two groups according to whether they provided care to bealthy or ill elders. Care providers for healthy and ill elders were ususally wives (28 and $43 \%$ ), son/daughters or daughters-in-law ( 51 and $40 \%$ ) and granddaughters ( 2 and $7.5 \%$ ). The three health services most frequently used by care providers were medical visits (65.2\%), friends or relatives $(41.2 \%)$ and alternative healers or non-traditional medicine (31.9\%). There were significant differences between bealthy and ill elders' care providers on depression $(t=3.195$, $p<0.01)$ and $E R S(t=1.955, p<0.05)$. Depression is associated with care providers age $(\beta=2.332)$, incomes $(\beta=-0.325)$, duration of care $(\beta=0.253)$, elders' gender $(\beta=0.143, p<0.003)$ and $E R S$ $(\beta=-1.984, p=0.001)$.

Discussion: Family is the main source of care for rural elders with lower socioeconomic income; wives and daughters assume the central role. They use medical and non-traditional resources. Care providers' depression is associated with characteristics of the elders, with availability of resources being a potential mediator.

Conclusion: Availability of social support and health service use are important for rural care providers, as resources to reduce the severity and presence of depression.

Key words: elders, care providers, rural, socioeconomic resources, social support, health services use, depression.

\section{INTRODUCCIÓN}

A medida que envejece la población, aumenta la demanda para la provisión de cuidados para los adultos mayores. Actualmente los familiares continúan siendo la principal fuente de cuidados, especialmente en las zonas rurales (1). La demanda de cuidados ha generado un cierto número de dificultades potenciales, incluyendo la sobrecarga de los servicios de salud (2). Por ejemplo, a medida que la esposa del adulto mayor también envejece y se vuelve frágil, la generación intermedia compuesta por los hijos se ve expuesta a una sobrecarga derivada de ayudar a sus padres, y al mismo tiempo cumplir con sus obligaciones laborales y paternales hacia sus propios hijos. Los estudios realizados sobre los cuidados prolongados brindados a los pacientes con demencia muestran una gran sobrecarga y agotamiento de los cuidadores (3), en cuyo resultado desempeña un rol impor- tante la red de apoyos sociales. En ese sentido, los estudios sugieren que a mayor apoyo social recibido, menor estrés sufren los cuidadores (4). Adoptar una estrategia adecuada para proveer apoyos sociales y laborales a los cuidadores puede tener un fuerte impacto en su salud. Los cuidadores de adultos mayores de zonas rurales son un grupo poco estudiado, por lo que podrían llegar a subestimarse sus reales necesidades en materia de apoyo social y demanda de servicios de salud. Se ha determinado en varios estudios, que la depresión es el problema de salud mental más frecuentemente referido por los cuidadores de pacientes con demencia o enfermedades mentales (5) y enfermedades físicas crónicas $(6,7)$. Por las anteriores razones se decidió conducir un estudio exploratorio en una zona rural, para determinar los perfiles de uso de servicios de salud, de apoyo social recibido, y la relación entre este último aspecto y la depresión entre los cuidadores de adultos 
Mediadores (apoyo social, servicios de salud, etc.)

Características socio-ambientales y económicas de los cuidadores (educación, ingresos, vivienda rural o urbana, género, etc.)

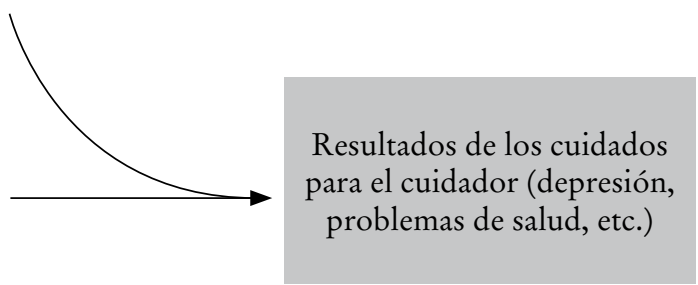

Figura 1. Esquema del marco teórico propuesto.

mayores. La hipótesis previa proponía que, de acuerdo con el modelo propuesto por Perlin (8), el resultado de los cuidados recibidos por los adultos mayores sería influido por las características socioeconómicas y ambientales de los cuidadores, a través de la mediación ejercida por los apoyos sociales brindados a estos últimos (Figura 1).

De acuerdo con la hipótesis prevista, se anticipaba que los resultados del estudio servirían para elucidar los efectos del apoyo social sobre el estado de salud y la depresión de los cuidadores, proveyendo datos objetivos para la implementación de programas de cuidados para los cuidadores en zonas rurales y/o urbanas, así como las diferencias entre estos. También se esperaba que los resultados del estudio fueran útiles para implementar redes de servicios de salud en zonas rurales que pudieran atender a los cuidadores de adultos mayores sanos y/o enfermos.

\section{SUJETOS Y MÉTODOS}

El estudio se realizó con la aprobación del comité de ética local, en dos medios sociales diferentes, uno urbano y otro rural. Todos los participantes del estudio dieron su consentimiento por escrito para participar del estudio. En la presente versión se analizan los datos correspondientes a la muestra rural.

\section{Descripción del campo.}

El estudio se realizó con una muestra de cuidadores residentes en la zona rural de la localidad de Chalicán (Provincia de Jujuy), que en febrero de 2008 sufrió una inundación que obligó a muchos de sus habitantes a evacuar el área. Esta pequeña localidad corresponde al departamento de Libertador General San Martín-Ledesma, con una superficie de 3249 $\mathrm{km}^{2}$ y una densidad poblacional de 23,3 habitantes por $\mathrm{km}^{2}$, de acuerdo con los datos del Censo 2001. El grupo de bajos ingresos socioeconómicos se definió como aquel con ingresos per cápita/familiar por debajo de la línea de pobreza (472,13 pesos argentinos) (Fuente: Indec) para el aglomerado Libertador General San Martín-Ledesma, mientras que el grupo de altos ingresos se definió como aquel con valores iguales o mayores al promedio de ingresos per cápita en el periodo 20072011, aglomerado por totales del país (3.047 pesos) (Fuente: BM). La localidad de Jujuy representa una zona agro-rural con cultivos no intensivos, con lento desarrollo socioeconómico y escasos recursos materiales. Los habitantes del lugar se dedican a labores agrícolas como el cultivo de caña de azúcar o cítricos, y a la pesca. Otros son beneficiarios de planes sociales, ya que escasean los puestos de trabajo. El estilo de vida en esta zona es despojado y simple, con la creencia de sus residentes de que el medio ambiente es seguro y los vecinos 
son mayormente confiables. No se aprecia un sentimiento de inseguridad caracterizado por el cierre compulsivo de puertas y ventanas, $y$ no se percibe la sensación de actitud defensiva ante la inminencia de un delito. Las personas son hospitalarias, ofrecen ayuda e información a los escasos visitantes, y en ocasiones comparten comidas. Las viviendas en general son humildes. No se realizan con frecuencia viajes fuera de la localidad, con excepción de traslados al centro urbano más próximo (Ledesma o Libertador General San Martín, a 25 $\mathrm{km})$ con fines administrativos, sanitarios o de compras, y cuenta con una población aproximada de 1.050 habitantes, una proporción de mayores de 65 años de 5,3\% (total provincial), índice de masculinidad general de 103.4, tasa de analfabetismo general del $18 \%$ y para los adultos mayores, del $24 \%$, índice de cobertura en salud del $56 \%$, porcentaje de hogares encabezados por mayores de 65 años del 15\% con casi $40 \%$ de hogares de tipo extendido.

\section{Sujetos}

Los censos poblacionales se obtuvieron de cifras oficiales del Indec. Los residentes adultos mayores se estratificaron en tres categorías: 60-69 $(\mathrm{n}=37), 70-79(\mathrm{n}=35)$ y más de $80(n=28)$. Se realizó una selección de los cuidadores mediante un método de muestreo aleatorio estratificado Se administró un cuestionario a los cuidadores $(n=145)$ de estos 100 adultos mayores, de los cuales respondieron 100 (tasa de respuesta del 68,9\%). Una parte de los entrevistados sufrió los efectos de una inundación en el año 2008, con sus secuelas psicológicas y económicas, que debió ser investigado con relación a su influencia en la depresión de los cuidadores para diferenciar su efecto con respecto al de las demás variables estudiadas (9).

\section{Instrumentos}

1. Escala de apoyo social funcional de DukeUNC (EAS).(10) Se trata de un cuestionario autoadministrado, que consta de 11 ítems y una escala de respuesta tipo Likert.(1-5) El rango de puntuación oscila entre 11 y 55 puntos y evalúa la fortaleza de las redes de apoyo social del sujeto. A menor puntuación, menor apoyo. El punto de corte se estableció en el percentil 15, que corresponde a una puntuación $<32$. Una puntuación igual o mayor a 32 indica un apoyo normal, mientras que menor a esa cifra señala un apoyo social percibido como bajo. Su consistencia interna oscila entre 0.88 y 0.79 .

2. Escala de redes sociales de Lubben (ERS) (11) consiste en 12 preguntas sobre redes familiares (6 preguntas) y redes de amigos (6 preguntas), evaluadas con una escala Likert de 0 (nunca) hasta 5 (siempre). Los puntajes van desde 0 como puntaje mínimo hasta 60 como puntaje máximo. Cuanto mayor el puntaje es mayor también el nivel de apoyo social. Un puntaje de $<20$ indica que la persona tiene una red social extremadamente limitada. Tiene buena validez y alta consistencia interna (Cronbach $\alpha: 0.83$ ).

3. Cuestionario de usos de servicios de salud (12) compuesto por cuatro dimensiones: uso de servicios de salud (sí o no), frecuencia (con cuánta frecuencia), objetivo (para quién), y utilidad (qué tan útiles), el puntaje osciló desde 0 a 2: $0=$ nunca usó los servicios de salud, 1 = usó los servicios; 2 = nunca usó los servicios pero los estima necesarios. La frecuencia se puntuó de 0 a 5: 0 = todos los días, o 5-7 veces por semana; 1 = una vez a 5 veces por semana; 2 = una vez por semana; 3 = más de una vez por mes pero menos de una vez por semana; $4=$ una vez por mes; $5=$ menos de una vez por mes. El propósito de la utilización se puntúo de 1 a 3; 1 = para el adulto mayor; 2 = para el propio cuidador; 3 = para ambos. La utilidad se puntuó de 1 a 3; 1 = no útil; 2 =parcialmente útil; 3 = muy útil. La escala mostró un alto acuerdo entre profesionales (kappa $=0.80$ ) $\mathrm{y}$ entre prescripciones $(\mathrm{kappa}=0.60)$, pero moderado para procedimientos diagnósti$\cos (\mathrm{kappa}=0.40)$.

4. Escala de depresión del Centro de Estudios Epidemiológicos (CED-D) (13) consiste de 20 ítems que puntúan de 0 a 3 . El 
puntaje total fue la suma de los puntajes individuales. Cuanto mayor el puntaje es más seria la depresión. Tiene un índice de confiabilidad en torno a $\alpha=0.85$ en población general y 0.90 en muestras clínicas, validez concurrente de $r=0.70$ con BDI, estructura factorial de cuatro factores interrelacionados: afecto depresivo aumentado, afecto positivo disminuido, actividad somática alterada, problemas interpersonales. El CES-D permite evaluar de manera confiable el nivel de depresión, en población general no clínica (screening) y grupos clínicos. Es breve, puede administrarse en entrevista o forma autónoma, tanto individual como colectiva.

\section{ANÁLISIS ESTADÍSTICO}

El universo estuvo compuesto por la población de la localidad investigada y la fórmula para el cálculo del tamaño muestral (14)

$$
S S=\frac{Z^{2 *}(p) *(1-p)}{c^{2}}
$$

donde:

$\mathrm{Z}=$ nivel de confianza (1.96 para nivel de confianza del $95 \%$ y error tipo I de $5 \%$ ) $\mathrm{p}=$ porcentaje encuestados con selección de respuesta, decimal ( 0.5 usado para la muestra necesaria)

$\mathrm{c}=$ intervalo de confianza, decimal $(0.04= \pm 4)$

Corrección para poblaciones finitas

$$
\text { nuevo SS }=\frac{\text { SS }}{1+\frac{\text { SS-1 }}{\text { pop }}}
$$

Donde pop = población

Para el análisis de los datos se empleó el paquete estadístico SPSS 16.0. Los cuidadores se dividieron en dos categorías: cuidadores de adultos mayores saludables (CAS) y de adultos mayores con enfermedades (CAE). El efecto de la inundación sobre la salud mental de los cuidadores afectados se evaluó mediante el coeficiente de correlación intraclase. Se estimó la frecuencia para variables categóricas como distribución de género, relación entre cuidador y adulto mayor, y la frecuencia de respuestas (verdadero-falso) para cada una de las preguntas de la escala de apoyo social funcional de Duke-UNC. Para las variables continuas como el puntaje total de la escala funcional de apoyo social (EAS), la escala de redes sociales (ERS) y la CES-D se utilizó un análisis descriptivo para explorar la media, desviación estándar, intervalo de confianza del $95 \%$ de la media. Se empleó test $\mathrm{t}$ de muestras independientes para comparar las diferencias de edad, tiempo dedicado al cuidado, los puntajes de la EAS, ERS y CES-D entre los cuidadores de ambos grupos. Las diferencias entre grupos para la distribución de frecuencias de género y utilización de servicios de salud se examinaron con test de Chi cuadrado (test $\chi^{2}$ ). La relación entre la CESD y las características demográficas de los cuidadores y adultos mayores se analizó en dos pasos por medio de regresión logística. En el primer paso (modelo 1) se introdujeron las características demográficas del cuidador y del adulto mayor, y se extrajeron los factores relacionados de manera significativa con la depresión, los cuales se introdujeron en el paso 2 del análisis, donde se analizó la significación estadística de la EAS, la ERS y los factores extraídos del paso 1 (modelo 2). Se definió como significación estadística un valor de $\alpha \leq 0.05$ (dos lados). El efecto diferencial de las consecuencias de la inundación sobre la salud mental de los cuidadores con respecto a las demás variables independientes estudiadas, se analizó con el coeficiente de correlación intraclase. Este análisis permite determinar si la depresión entre los cuidadores que sufrieron la inundación es mayor que la que se encuentra entre los que no la sufrieron. Dicho en otros términos, permite distinguir el efecto de la inundación sobre la depresión con respecto a las demás variables. Su valor oscila entre 0 (ningún efecto de la inundación sobre la depresión) y 1 (la depresión se debe casi exclusivamente a los efectos de la inundación sobre la salud mental). 


\section{RESULTADOS}

Las características demográficas de los participantes (cuidadores y adultos mayores) se describen en la Tabla 1. Los cuidadores ( $\mathrm{n}=100$ tuvieron una edad media de $51.3 \pm 12.2$ años, en tanto que para los adultos mayores $(\mathrm{n}=100)$ fue de 75.8 \pm 1.9 . El test t para muestras independientes mostró que las edades de los cuidadores de adultos mayores sanos (AMS) y de adultos mayores enfermos (AME) difirió de manera significativa entre ambos ( $\mathrm{t}=-2.17, \mathrm{p}<0.05)$ mientras que las edades de los adultos mayores, sanos y enfermos, no difirieron entre sí $(\mathrm{t}=-0.163, \mathrm{p}>0.05)$. El test de $\chi^{2}$ no demostró diferencias significativas en la distribución de género entre los adultos mayores, tanto sanos como enfer- mos, $y$ sus cuidadores $(C)\left(\chi^{2}=0.054, p>0.57 y\right.$ $\chi^{2}=0.095, p>0.59$ respectivamente). Los AME $(\mathrm{n}=64)$ recibían cuidados de la esposa $(43 \%)$, hijos $(7,5 \%)$ e hijos y nueras (40\%). La mayoría de cuidadores viven junto con el adulto mayor cuidado, tanto sano $(93,6 \%)$ como enfermo $(81 \%)$, a menudo en una vivienda familiar. La mayoría de los cuidadores trabajan la tierra $(53,2 \%)$ o trabajan en el hogar $(28,8 \%)$, tienen en promedio cerca de 3 años de escolarización, un poco más de la tercera parte tienen ingresos por debajo de la línea de pobreza y no disponen de ahorros bancarios. No hubo diferencias significativas entre la residencia de los cuidadores $\left(\chi^{2}=3.163, \mathrm{p}>0.05\right)$, estado civil $\left(\chi^{2}=2.467, \mathrm{p}>0.05\right)$, ocupación $\left(\chi^{2}=8.489, \mathrm{p}>0.05\right)$, y tiempo dedicado a los cuidados $(t=-1.375, p>0.05)$ entre los grupos

Tabla 1. Características demográficas de cuidadores (C) y adultos mayores (AM) en una zona rural.

\begin{tabular}{|c|c|c|c|c|}
\hline \multirow{2}{*}{ Características } & \multicolumn{2}{|c|}{ AMS $(\mathrm{N}=36)$} & \multicolumn{2}{|c|}{$\operatorname{AME}(\mathrm{N}=64)$} \\
\hline & $\mathrm{C}$ & $\mathrm{AM}$ & $\mathrm{C}$ & $\mathrm{AM}$ \\
\hline Edad (media $\pm \mathrm{DE})$ años & $55.4 \pm 4.3$ & $76.4 \pm 2.3$ & $44.2 \pm 2.9$ & $75.1 \pm 2.7$ \\
\hline \multicolumn{5}{|l|}{ Género } \\
\hline Femenino & 30 & 30 & 61 & 61 \\
\hline Masculino & 6 & 6 & 3 & 3 \\
\hline \multicolumn{5}{|l|}{ Relación C-AM } \\
\hline Esposa & $10(28 \%)$ & & $27(43 \%)$ & \\
\hline Hijos y nueras & $18(51 \%)$ & & $25(40 \%)$ & \\
\hline Nietos & $1(2 \%)$ & & $5(7.5 \%)$ & \\
\hline Otros familiares & $7(19 \%)$ & & $6(9.5 \%)$ & \\
\hline \multicolumn{5}{|l|}{ Residencia (n \%) } \\
\hline Vivienda familiar & $34(93.6 \%)$ & & $52(81 \%)$ & \\
\hline \multicolumn{5}{|l|}{ Educación (años) } \\
\hline$<$ 1año & $11(32 \%)$ & & $31(48 \%)$ & \\
\hline 1-5 años & $13(35 \%)$ & & $19(30 \%)$ & \\
\hline 6-9 años & $7(19 \%)$ & & $6(9 \%)$ & \\
\hline 9-12 años & $3(8 \%)$ & & $5(7.5 \%)$ & \\
\hline Sin datos & $2(6 \%)$ & & $3(5.5 \%)$ & \\
\hline \multicolumn{5}{|l|}{ Ingresos } \\
\hline No cubre los gastos & $6(17 \%)$ & & $18(28.3 \%)$ & \\
\hline Tiene lo suficiente pero no mas & $13(35 \%)$ & & $23(35.9 \%)$ & \\
\hline Lo suficiente y algo extra & $13(37 \%)$ & & $13(20.7 \%)$ & \\
\hline Siempre me sobra & $2(5 \%)$ & & $4(5.7 \%)$ & \\
\hline Datos faltantes & $2(6 \%)$ & & $6(9.4 \%)$ & \\
\hline \multicolumn{5}{|l|}{ Estado civil } \\
\hline Casado & $31(86 \%)$ & & $52(81 \%)$ & \\
\hline Viudo & $2(6 \%)$ & & $6(11 \%)$ & \\
\hline Separado & $1(4 \%)$ & & $7(6 \%)$ & \\
\hline Soltero & $1(4 \%)$ & & $1(2 \%)$ & \\
\hline $\mathrm{C}$ viviendo con $\mathrm{AM}$ (sí, n \%) & $25(70 \%)$ & & $49(77 \%)$ & \\
\hline Tiempo de cuidados $[\mathrm{hr} / \mathrm{sem}]($ media $\pm \mathrm{DS})$ & $20.1 \pm 11.8$ & & $30.3 \pm 21.8$ & \\
\hline $\mathrm{CCI}$ & 0.013 & & 0.018 & \\
\hline
\end{tabular}

CCI: coeficiente de correlación intraclase 
de cuidadores de AMS y AME. El coeficiente de correlación intraclase promedio entre cuidadores de AMS y AME fue de 0.015 (muy bajo), lo que indica que solo el $1,5 \%$ de la incidencia de la depresión entre los cuidadores fue explicado por las consecuencias de la inundación.

\section{USO DE LOS SERVICIOS DE SALUD, APOYO SOCIAL Y EVALUACIÓN DE LA DEPRESIÓN}

Como se observa en la Figura 2, el servicio de salud más usado fue el de visitar a un médico (SU3) $(65,2 \%)$, seguido de recurrir a los amigos para obtener apoyo adicional (SU18)
$(41,2 \%)$ y buscar ayuda por parte de los practicantes informales (curanderos o manosantas) (SU16) (31,9\%).

La Tabla 2 muestra el uso de estos tres servicios (recurrir al médico, amigos o practicantes informales) entre los cuidadores. La mayoría de los cuidadores $(72,2 \%)$ no ve al médico muy seguido ( $1 \mathrm{o}<1 \mathrm{vez} / \mathrm{mes})$. Cerca de la mitad visita médicos $(45,6 \%)$ mayormente para el adulto mayor, y a más de la mitad $(61,0 \%)$ le resulta muy útil. El cuidador de AME mostró una mayor tendencia a recurrir a los servicios de médicos, coincidente con los resultados de otros estudios $\left(\chi^{2}=6.482, p<0.05\right)(15)$. El uso de otros servicios no difirió significativamente entre ambos grupos $(\mathrm{p}>0.68)$.

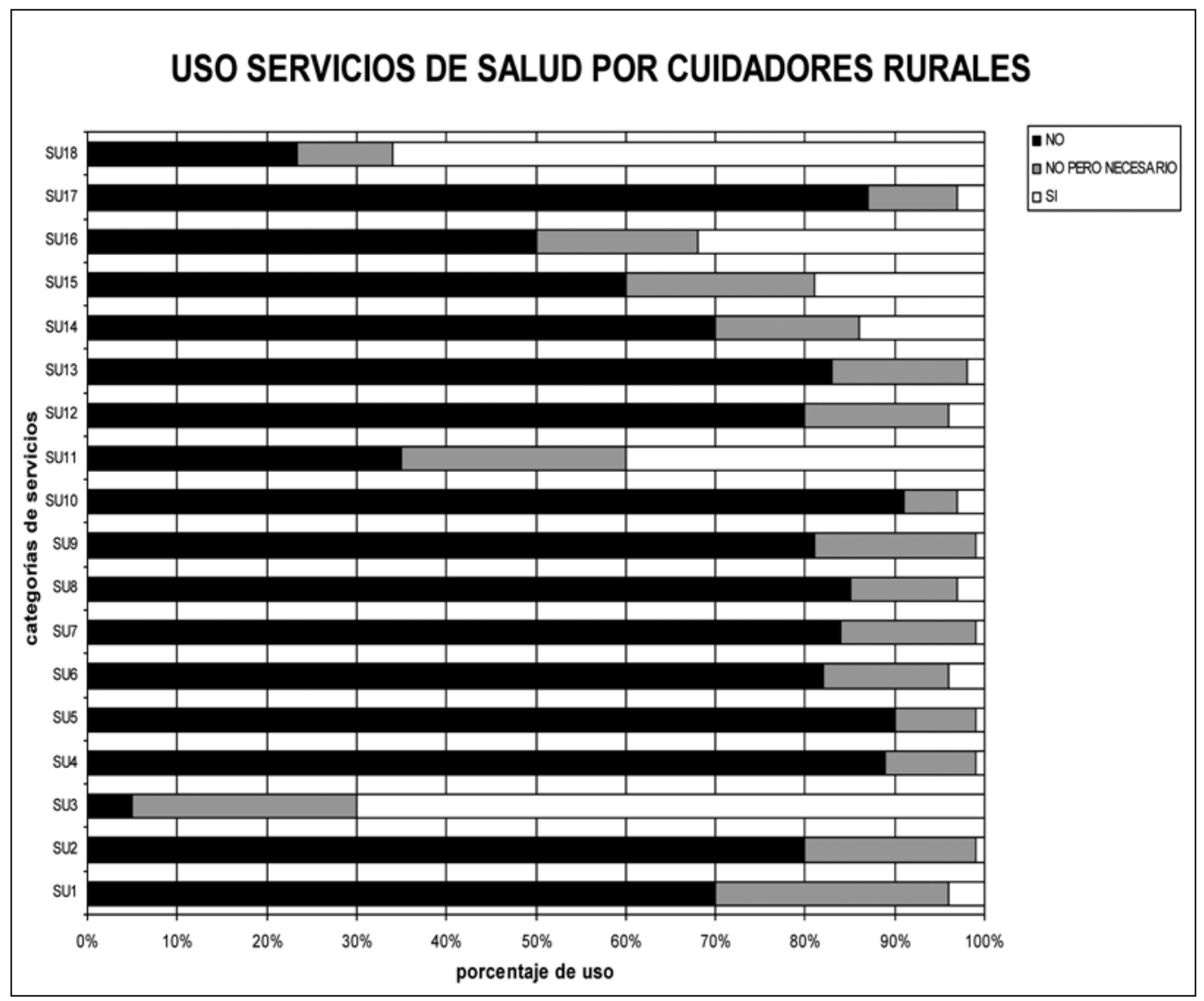

Nota: SU1 = cuidados de día; SU2 = cuidadores domiciliarios; SU3 = visita médica; SU4 = internación domiciliaria; SU5 = acompañamiento pago; $\mathrm{SU} 6$ = mejoras domiciliarias; $\mathrm{SU} 7$ = asistente domiciliaria; $\mathrm{SU} 8=$ descanso; $\mathrm{SU} 9=$ trabajador social; $\mathrm{SU} 10=$ entrega de comidas a domicilio; SU11 = transporte; SU12 = asesoramiento de salud mental; SU13 = consejo legal; SU14 = consejo financiero; SU15 = curanderos o sanadores; SU16 = médicos; $\mathrm{SU} 17$ = grupos de apoyo; SU18 = amigos o parientes. 
Tabla 2. Uso de tres servicios comunes de salud entre cuidadores en área rural.

\begin{tabular}{lllllllllllllll}
\hline & \multicolumn{3}{c}{ Frecuencia de uso (\%) } & \multicolumn{4}{c}{ Para quién (\%) } & \multicolumn{3}{c}{ Utilidad (\%) } \\
& 0 & 1 & 2 & 3 & 4 & 5 & C & AM & ambos & 1 & 2 & 3 \\
Visita al médico & 2.5 & 2.9 & 5.7 & 16.7 & 7.9 & 64.3 & 45.6 & 19.9 & 34.5 & 1.7 & 37.3 & 61.0 \\
Buscar ayuda de amigos & 2.5 & 6.1 & 1.5 & 5.1 & 4.8 & 81.0 & 57.2 & 6.1 & 36.7 & 1.4 & 45.6 & 53.0 \\
Ver a practicante informal & 0 & 1.5 & 5.4 & 6.4 & 8.8 & 77.9 & 35.2 & 36.8 & 27.9 & 2.1 & 51.9 & 45.9 \\
\hline
\end{tabular}

Frecuencia: $0=$ diaria, $5-7$ días $/$ semana; $1=>1 \mathrm{vez} /$ semana, $<5$ días $/$ semana; $2=1 \mathrm{vez} / \mathrm{semana} ; 3=<1 \mathrm{vez} / \mathrm{semana},>1 \mathrm{vez} / \mathrm{mes} ; 4=$ una $\mathrm{vez} / \mathrm{mes} ; 5=<1 \mathrm{vez} / \mathrm{mes}$. Utilidad: $1=$ nada útil; $2=$ algo útil; $3=$ muy útil $\mathrm{C}=$ cuidador; $\mathrm{AM}=$ adulto mayor.

Los puntajes de las respuestas de la EAS se exhiben en la Tabla 3. No hubo diferencias significativas entre ambos grupos en la EAS $(\mathrm{t}=0.627, \mathrm{p}>0.41)$. Los cuidadores de AME puntuaron más alto en la escala de depresión y menor en la de ERS que los cuidadores de AMS $(\mathrm{t}=3.195, \mathrm{p}<0.01$ y $\mathrm{t}=1.955, \mathrm{p}>0.05$ respectivamente). El análisis de correlación de Pearson encontró una relación positiva entre las EAS y ERS ( $\mathrm{r}=0.369, \mathrm{p}<0.001)$, sugiriendo la consistencia de ambas escalas para la evaluación de apoyo social, y también encontró una correlación negativa entre la CES-D y EAS ( $\mathrm{r}=-0.217, \mathrm{p}=0.001)$, y la CE-D y ERS $(\mathrm{r}=-0.418, \mathrm{p}<0.001)$. Estos resultados sugieren que la severidad de la depresión disminuye a medida que aumenta el grado de apoyo social.

La comparación entre las EAS, ERS y CES-D se muestra en la Tabla 4 donde se observa que las diferencias son más significativas entre los cuidadores de AMS y AME en las escalas de redes sociales y depresión, en tanto no hay tantas diferencias en apoyo social.

\section{RELACIONES ENTRE LA DEPRESIÓN Y LAS CARACTERÍSTICAS DE LOS CUIDADORES Y ADULTOS MAYORES}

Como se muestra en la Tabla 5, el análisis de regresión logística tomando como variable dependiente la presencia de depresión en el cuidador, mostró que las variables confundidoras, incluyendo edad y género de AM y cuidadores, ingresos, residencia y tiempo dedicado a cuidados, no obtuvieron diferencias estadísticamente significativas, con excepción del tiempo de cuidados y el nivel de educa- ción del cuidador con una relación inversa, es decir, a menor nivel educativo, es mayor la severidad de la depresión (modelo 1). Tomando en cuenta el efecto de las variables independientes se comprobó que hubo una relación significativa entre la edad e ingresos de los cuidadores y el tiempo de cuidados, los valores de redes sociales y uso de servicios de salud con la severidad de la depresión (modelo 2 en Tabla 5).

\section{DISCUSIÓN}

La hipótesis planteada al comienzo del estudio fue que no solo el resultado de los cuidados recibidos por los adultos mayores sería influido por las características socioeconómicas y ambientales de los cuidadores, sino que estas también ejercerían una influencia sobre la presencia de depresión en el cuidador; a través de la mediación ejercida por los apoyos y recursos sociales, dentro del contexto de una comunidad rural. Los resultados preliminares informan que: 1) la mayoría de cuidadores usan recursos médicos durante el cuidado del anciano, tanto para sí mismos como para el AM; 2) la depresión difiere significativamente entre los cuidadores de ancianos sanos y enfermos; 3) la severidad de la depresión está relacionada con las características de los cuidadores, como su nivel de ingresos, edad, tiempo dedicado a los cuidados; 4) la mayor parte de los cuidadores fueron esposa e hijas, consistente con las tradiciones rurales (16) en tanto que los hijos trabajan fuera del hogar y disponen de mayores redes sociales que reducen la depresión; 5) a menor nivel de ingresos aumenta el riesgo de depresión, por aumento 
Tabla 3. Promedio de respuesta a las preguntas en la EAS.

\begin{tabular}{lc}
\hline Preguntas & Promedio de frecuencias $(\%)$ \\
\hline Recibo visitas de amigos y familiares & 28.7 \\
Recibo amor y afecto & 27.3 \\
Recibo ayuda en asuntos relacionados con mi casa & 25.2 \\
Recibo elogios y reconocimiento cuando hago bien mi trabajo & 28.4 \\
Tengo oportunidad de hablar con alguien acerca de mis problemas & 29.0 \\
en el trabajo o en la casa & \\
Tengo oportunidad de hablar con alguien de mis problemas familiares o personales & 27.6 \\
Tengo oportunidad de hablar con alguien de problemas económicos & 26.3 \\
Recibo invitaciones para distraerme y salir con otras personas & 27.3 \\
Recibo consejos útiles acerca de cosas importantes en la vida & 29.4 \\
Recibo ayuda cuando estoy enfermo en cama & 28.5 \\
\hline
\end{tabular}

Tabla 4. Comparación entre los puntajes de escala apoyo social, redes sociales y CES-D.

\begin{tabular}{|c|c|c|c|c|c|c|}
\hline & \multicolumn{4}{|c|}{ Puntajes de Escalas } & \multicolumn{2}{|c|}{ CAS/CAE } \\
\hline & \multicolumn{2}{|l|}{ CAS $(n=36)$} & \multicolumn{2}{|l|}{$\operatorname{CAE}(\mathrm{n}=64)$} & \multirow{2}{*}{$\mathrm{t}$} & \multirow{2}{*}{$p$} \\
\hline & Media (DE) & $95 \%$ IC & Media (DE) & $95 \%$ IC & & \\
\hline Escala apoyo social & $16.8(2.8)$ & $15.3-17.9$ & $16.2(2.6)$ & $15.3-17.1$ & 0.538 & 0.549 \\
\hline Escala redes sociales & $26.9(5.3)$ & $24.2-29.4$ & $23.9(6.9)$ & $21.2-26.2$ & 1.855 & 0.002 \\
\hline CES-D & $15.8(6.1)$ & $13.2-17.9$ & $18.9(7.1)$ & $17.9-21.1$ & -3.417 & 0.001 \\
\hline
\end{tabular}

CAS: cuidadores adultos sanos, CAE: cuidadores adultos enfermos

Tabla 5. Regresión logística para variable dependiente CES-D.

\begin{tabular}{|c|c|c|c|c|c|c|c|c|c|c|}
\hline & \multicolumn{5}{|c|}{ Modelo 1} & \multicolumn{5}{|c|}{ Modelo 2} \\
\hline & \multicolumn{2}{|c|}{$\begin{array}{l}\text { Coeficientes no } \\
\text { estandarizados }\end{array}$} & \multirow{2}{*}{$\begin{array}{l}\text { Coef. } \\
\text { Beta }\end{array}$} & \multirow{2}{*}{$\mathrm{T}$} & \multirow{2}{*}{$(p)$} & \multicolumn{2}{|c|}{$\begin{array}{l}\text { Coeficientes no } \\
\text { estandarizados }\end{array}$} & \multirow{2}{*}{$\begin{array}{l}\text { Coef. } \\
\text { Beta }\end{array}$} & \multirow{2}{*}{$\mathrm{T}$} & \multirow{2}{*}{$(p)$} \\
\hline & B & SE & & & & B & SE & & & \\
\hline Edad & 0.134 & 0.043 & 0.316 & 1.901 & 0.022 & 0.143 & 0.154 & 0.232 & 2.435 & 0.096 \\
\hline Ingresos AM & -2.921 & 0.834 & -3.215 & 3.267 & 0.055 & -2.543 & 0.566 & -3.445 & 2.553 & 0.011 \\
\hline Residencia AM & 2.468 & 1.693 & 0.153 & 2.093 & 0.072 & 2.766 & 1.325 & 0.214 & 1.943 & 0.012 \\
\hline Género AM & 1.438 & 1.215 & 0.215 & 1.745 & 0.067 & 1.332 & 1.355 & 0.143 & 1.332 & 0.003 \\
\hline Edad AM & 0.153 & 0.047 & 0.436 & 2.943 & 0.054 & 0.243 & 0.094 & 0.225 & 2.444 & 0.056 \\
\hline Tiempo cuidados & 0.05 & 0.037 & 0.167 & 2.998 & 0.008 & 0.254 & 0.133 & 0.253 & 2.423 & 0.001 \\
\hline \multicolumn{11}{|l|}{ F. mediadores } \\
\hline Edad C & -3.232 & 0.523 & -2.642 & 3.834 & 0.032 & 4.126 & 0.321 & 2.332 & 3.790 & 0.007 \\
\hline Ingreso C & 2.923 & 1.933 & 0.184 & 2.374 & 0.073 & 2.422 & 1.547 & -0.325 & 2.833 & 0.008 \\
\hline Residencia C & 1.922 & 0.038 & 0.743 & 2.394 & 0.043 & 1.643 & 0.673 & -0.654 & 2.098 & 0.044 \\
\hline Género C & 0.023 & 0.936 & 0.845 & 2.184 & 0.005 & 0.311 & 0.744 & 0.758 & 1.276 & 0.004 \\
\hline Educación C & -0.324 & 1.326 & 0.887 & .2 .365 & 0.002 & 1.655 & 0.632 & 0.844 & -1.443 & -0.001 \\
\hline EAS & & & & & & 0.265 & 0.098 & 1.065 & 4.934 & 0.001 \\
\hline ERS & & & & & & -0.255 & 0.934 & -1.984 & 3.654 & 0.001 \\
\hline Uso servicios salud & & & & & & & & & & \\
\hline
\end{tabular}

C: cuidadores, AM: adultos mayores; F: mediadores: factores mediadores, Coef Beta: coeficiente beta 
de la dependencia económica y carga de trabajo y menores oportunidades de mantener vínculos sociales (17); 6) la mayoría de cuidadores visitan al médico para los problemas de salud, y en ausencia de causas definidas de enfermedad, especialmente crónicas, se recurre a curadores informales; 7) los cuidadores de AMS tenían menos depresión que los cuidadores de AME, ya que estos últimos demandan una mayor carga de cuidados, algo coincidente con otros estudios.(18-21)

Además de confirmar la hipótesis inicial sobre aumento de depresión en cuidadores, asociada a características socioambientales y mediada/moderada por redes sociales y uso de servicios asistenciales, se sugieren modos de intervención para reducir la depresión en este grupo poblacional: 1) promover redes sociales alternativas para los cuidadores y servicios sociales de ayuda para sus tareas; 2) mantener vigilancia epidemiológica para estos cuidadores (edad promedio 45 años) para prevenir depresión y otras enfermedades asociadas al estrés (hipertensión, tabaquismo, alcoholismo); 3) ya que la mayoría de los cuidadores estaban casados, deberían recibir ayuda económica y/o social para compensar sus esfuerzos y mantener la cohesión familiar; 4) adaptar las instrucciones de acuerdo al bajo nivel educativo de los cuidadores (primario incompleto en su mayoría), lo que de por sí representa un obstáculo adicional para construir redes sociales y mantener empleos formales; 5) en consonancia con lo anterior, sería conveniente fomentar planes educativos informales asociados a la ayuda social para compensar la instrucción faltante; 6) debido al escaso uso de servicios informales como transporte, visitas al médico o amigos y parientes, así como los escasos períodos de descanso para los cuidadores con más depresión, debería facilitarse este tipo de recursos con visitas domiciliarias programadas de diferentes agentes de salud y servicios sociales; 7) enseñar estrategias de afrontamiento de los problemas basadas en reconocimiento de obstáculos y reclamo de soluciones, antes que en la negación de los mismos, que terminan derivando en cuadros depresivos de difícil resolución; 8) finalmente, enseñar técnicas de cuidado tanto de AMS como AME, preferentemente en terreno, teniendo en cuenta la escasa experiencia y reducidos recursos cognitivos y ambientales de los cuidadores. Limitaciones: Reducido tamaño muestral, población rural que impide generalizar los resultados a otros ámbitos, estudio transversal que impide esbozar relaciones causales.

\section{CONCLUSIÓN}

Los cuidadores rurales de AMS y AME representan un grupo poblacional subestimado y poco estudiado, con alta carga de trabajo y repercusiones físicas y psicológicas (depresión) no siempre detectadas, por lo que se requieren más estudios para explicar y explorar el apoyo social que reciben y su salud mental, así como para implementar planes socioambientales preventivos que les permitan mantener su autonomía y salud.

\section{REFERENCIAS}

5. Wenger GC, Scott A, Seddon D. The experience of caring for older people with dementia in a rural area: using services. Aging E Mental Health. 2002; 6(1): 30-38.

6. Freund D, Smeeding TM. The future costs of health care in ageing societies: is the glass half full or half empty? En: Tuljapurkar S, Ogawa N, Gauthier NH (eds.). Ageing in advanced industrial states. (Springer); 2010. pp. 173-193.

7. Benjumea C. Cuidado familiar en condiciones crónicas. Una aproximación a la literatura. Teto Contexto Enferm. 2004; 13(1): 137-146.

8. Molina JM, Iáñez M, Iáñez B. El apoyo social como modulador de la carga del cuidador de enfermos de Alzheimer. Psicol Salud. 2005; 15(1): 33-43.

9. Domínguez JA, Ruiz M, Gómez I, Gallego E, Valero J, Izquierdo MT. Ansiedad y depresión en cuidadores de pacientes dependientes. Semergen. 2012; 38(1): 16-23.

10. Dueñas E, Martínez MA, Morales B, Muñoz C, Viáfara AS, Herrera JA. Síndrome del 
cuidador de adultos mayores discapacitados y sus implicaciones psicosociales. Colomb Méd. 2006; 37(Supl 1): 31-38.

11. García-Calvente MDM, Mateo-Rodríguez I, Eguiguren AP. El sistema informal de cuidados en clave de desigualdad. Gac Sanit. 2004; 18: 132-139.

12. Pearlin LI, Mullan JT, Semple SJ, Skaff MM. Caregiving and the stress process: an overview of concepts and their measures. Gerontologist. 1990; 30(5): 583-594.

13. Gaborit M. Desastres y trauma psicológico. Pensamiento psicológico. 2010; 2(7): 15-39.

14. Broadhead WE, Gehlbach SH, de Gruy FV, Kaplan BH. The Duke-UNC Functional Social Support Questionnaire: measurement of social support in family medicine patients. Med Care. 1988; 26: 709-23.

15. Lubben JE. Assessing social networks among elderly populations. Fam Comm Health. 1988; 11: 42-52

16. Lubeck DP, Hubert HE. Self-report was a viable method for obtaining health care utilization data in community-dwelling seniors. J Clin Epidemiol. 2005; 58(3): 286-290.

17. Radloff LS. The CES-D scale: A self report depression scale for research in the general population. Appl Psychol Meas. 1977; 1: 385401.

18. Robinson L, Neutons JJ. Research techniques for the bealth sciences. New York: Macmillan; 1987.

19. Alvarado R, Numhauser J, Luisa G. Cuadros depresivos y utilización de servicios de salud en mujeres de la isla de Chiloé. Rev Chil Salud Pública 2006; 10(1): 35-46.

20. Vidal Gutiérrez D, Zaval Gutiérrez M, Castro Salas M, Quiroga López P, Klaasen Pinto G. El significado del paciente con demencia para el cuidador en una comunidad urbana y rural. Rev Servicio Social. 1999; 1(2): 1-10.

21. Grant JS, Bartolucci AA, Elliot TR, Giger JN. Socio-demographic, physical, and psychosocial characteristics of depressed and non-depressed family caregivers of stroke survivors. Brain Injury. 2000; 14(12): 10891100.

22. Nieboer AP, Schulz R, Matthews KA, Scheier MF, Ormel J, Lindenberg SM. Spousal caregivers' activity restriction and depression: A model for changes over time. Social Sci Med. 1998; 47(9): 1361-1371.

23. Schrag A, Hovris A, Morley D, Quinn $\mathrm{N}$, Jahanshahi $\mathrm{M}$. Caregiver-burden in Parkinson's disease is closely associated with psychiatric symptoms, falls, and disability. Parkinsonism Relat Disord. 2006 Jan; 12(1): 35-41.

24. Minh HV, Huong DL, Wall S, Chuc N, Byass P. Multilevel analysis of covariation in socioeconomic predictors of physical functioning and psychological well-being among older people in rural Vietnam. BMC Geriatrics. 2010, 10: 7.

25. Vandervoort D, Skorikov V. Physical health and social network characteristics as determinants of mental health across cultures. Current Psychol. 2006; 21(1): 50-67. 